\title{
Modeling and Analysis of Rayleigh Fading Channels using Stochastic Network Calculus
}

\author{
Huimin She ${ }^{1}$, Zhonghai $\mathrm{Lu}^{1}$, Axel Jantsch ${ }^{1}$, Dian Zhou ${ }^{2}$, Li-Rong Zheng ${ }^{1}$ \\ ${ }^{1}$ iPack VINN Excellence Center, School of ICT, Royal Institute of Technology (KTH), Sweden \\ ${ }^{2}$ State Key Lab of ASIC \& System, Dept. of Microelectronics, Fudan University, China \\ Email: \{huimin, zhonghai, axel, lirong\}@kth.se, zhoud@fudan.edu.cn
}

\begin{abstract}
Deterministic network calculus (DNC) is not suitable for deriving performance guarantees for wireless networks due to their inherently random behaviors. In this paper, we develop a method for Quality of Service (QoS) analysis of wireless channels subject to Rayleigh fading based on stochastic network calculus. We provide closed-form stochastic service curve for the Rayleigh fading channel. With this service curve, we derive stochastic delay and backlog bounds. Simulation results verify that the bounds are reasonably tight. Moreover, through numerical experiments, we show the method is not only capable of deriving stochastic performance bounds, but also can provide guidelines for designing transmission strategies in wireless networks.
\end{abstract}

\section{INTRODUCTION}

Network calculus is a theory dealing with performance guarantees in packet switching networks [1], [2], [3], [4], [5]. With the abstraction of arrival curve for traffic flows and service curve for network elements, it has been widely applied in communication networks for performance analysis.

In general, network calculus has been developed in two tracks: deterministic network calculus (DNC) and stochastic network calculus (SNC). The DNC generally considers the worst-case performance analysis through deterministic arrival curve and service curve. Recently, it has been extended and applied for worst-case performance analysis of sensor networks by several researchers [6] [7]. However, since data communication in wireless networks is unstable and irregular, it is very difficult even impossible to find the deterministic performance bounds. To incorporate nondeterministic service provisioning, the performance bounds have to be complemented with certain violation probabilities. SNC is such a tool which can be employed in the design of wireless networks to provide stochastic service guarantees.

In this paper, we propose a network calculus based approach for Quality of Service (QoS) analysis of wireless channels subject to Rayleigh fading. We develop a stochastic service curve model for the Rayleigh fading channel. Based on this model, we provide formulas to derive the probabilistic delay and backlog bounds in the cases of deterministic and stochastic arrival curves. The simulation results verify that the tightness of the bounds are good. Furthermore, through numerical experiments, we show the analysis method is not only capable of deriving performance bounds with corresponding violation probabilities, but also can provide guidelines for designing transmission strategies of wireless networks.
The rest of this paper is organized as follows. Section II includes related work. Section III presents the model of Rayleigh fading channel and the stochastic service curve. Section IV contains derivation of performance bounds. Simulations and numerical experiments are implemented in Section V. Finally, conclusions and future work are given in Section VI.

\section{RELATED WORK}

In general packet switching networks, network calculus provides the means to deterministically reason about timing properties and resource requirements. Systematic accounts of network calculus can be found in books [3] [4].

DNC is recently extended and applied for performance analysis and resource dimensioning of WSNs by several researchers [6] [7] [8] [9] [10] [11]. In [6], Schmitt et al. firstly applied network calculus to sensor network and proposed a generic framework for performance analysis of WSNs with various traffic patterns. They further extended the general framework to incorporate computational resources besides the communication aspects of WSNs [8]. In [7], Anis et al. proposed a methodology for the modeling and worst-case dimensioning of cluster-tree sensor networks. They derived plug-and-play expressions for the end-to-end delay bounds, buffering and bandwidth requirements as a function of the WSN cluster-tree and traffic characteristics. In [10], the authors presented a method for computing the worst-case delays, buffering and bandwidth requirements while assuming that the sink node can be mobile.

Research on SNC has the potential of providing insights into stochastic service guarantees of packet networks [12] [13] [14] [15]. In [13], Burchard et al. introduced the concept of statistical service curves as a probabilistic bound on the service received by an aggregation of flows or a single flow. Ciucu et $a l$. [12] extended the stochastic network calculus by providing a network service curve formulation which is capable of calculating stochastic end-to-end delay and backlog bounds for a number of arrival and service distributions. In [16], Jiang and Emstad proposed a server model to facilitate stochastic service guarantee analysis and address the challenges of delay guarantee, backlog guarantee, output characterization and concatenation property. There are a lot of works providing theoretic fundamentals of stochastic network calculus, but few of them study the problem of mapping the theory to a specific application. In [15], the authors presented a method for 
analyzing wireless channels by modeling channels as Markov chains. And they evaluated the delay tail distribution. However, they did not give closed-form service curves for the channel. Our work differs from previous work and mainly contributes in the following way: we propose a method for stochastic QoS analysis of the Rayleigh fading wireless channel. Moreover, a closed-form service curve for the channel is derived.

\section{Modeling of the Rayleigh Fading Channel}

\section{A. Channel model}

Fig. 1 shows the system model of a discrete-time flat-fading Rayleigh channel [17], which can be expressed by,

$$
Y=\left|h_{t}\right| e^{j \varphi} X+Z
$$

where $X$ and $Y$ are the channel input and output, respectively, $Z$ is the independent and identically distributed (i.i.d.) Gaussian noise; $\left|h_{t}\right| e^{j \varphi}$ is a complex channel gain with amplitude $\left|h_{t}\right|$ which is a random variable with a Rayleigh distribution, and phase $\varphi$ is uniformly distributed in $[0,2 \pi)$.

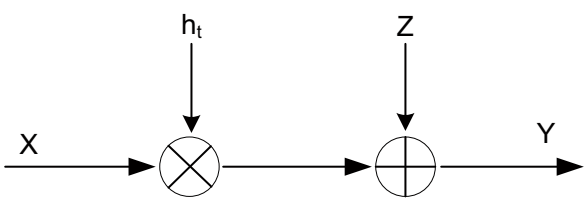

Fig. 1. System model of a fading channel.

Some assumptions about this model are: 1) Channel distribution information (CDI) is available both at the transmitter and receiver; 2) Channel state information (CSI) is only available at the receiver; 3) it is a slow-fading channel, i.e., the channel state does not change during the transmission of a packet.

Let $P_{t x}, W$, and $N_{0}$ denote the average transmission power, channel bandwidth, and power spectral density of the noise, respectively. The channel capacity can be expressed as,

$$
C=W \log _{2}\left(1+10^{\gamma_{t} / 10}\right)=W \log _{2}\left(1+\frac{P_{t x}\left|h_{t}\right|^{2}}{W N_{0}}\right) .
$$

Since the transmitter does not know the instantaneous signalto-noise ratio (SNR) $\gamma_{t}$ (in $d B$ ), it can not adjust its transmission power according to the channel condition. Hence, the transmission data rate can be considered as a constant regardless of the SNR of the received signal and there is probability of outage. For a transmission data rate $R$, the outage probability of a Rayleigh fading channel can be expressed by,

$$
\begin{aligned}
p_{\text {out }}(R) & =\operatorname{Pr}\{C<R\} \\
& =\operatorname{Pr}\left\{W \log _{2}\left(1+\frac{P_{t x}\left|h_{t}\right|^{2}}{N_{0} W}\right)<R\right\} \\
& =\operatorname{Pr}\left\{\left|h_{t}\right|^{2}<\left(2^{R / W}-1\right) \cdot \frac{N_{0} W}{P_{t x}}\right\},
\end{aligned}
$$

where the channel gain $\left|h_{t}\right|$ has a Rayleigh distribution with probability density function (pdf)

$$
f(x)=x \cdot \exp \left(-\frac{x^{2}}{2}\right) .
$$

By the transformation theorem, $\left|h_{t}\right|^{2}$ has an exponential distribution with pdf,

$$
g(x)=\frac{1}{2} \exp \left(-\frac{x}{2}\right) .
$$

Therefore, the outage probability is derived as,

$$
p_{\text {out }}(R)=1-\exp \left(\frac{1-2^{R / W}}{2 \cdot 10^{S N R / 10}}\right),
$$

where $\mathrm{SNR}=10 \log _{10}\left[P_{t x} /\left(N_{0} W\right)\right]$ denotes the signal-tonoise-ratio in $d B$.

\section{B. Stochastic service curve}

Since the channel capacity $C$ is a random, deterministic service curve is not suitable for capturing its characteristics. Thus, we use the stochastic service curve to characterize the service capability of the channel, which is described by two parameters: the data transmission rate $R$, and the error function $\epsilon$. According to the previous analysis of the Rayleigh channel, it can be modeled by the stochastic service curve $\langle\beta(t), \epsilon\rangle$, where

$$
\beta(t)=R \cdot t \quad \text { and } \quad \epsilon(R)=1-\exp \left(\frac{1-2^{R / W}}{2 \cdot \mathrm{SNR}}\right) .
$$

The violation probability function $\epsilon$ defines the outage probability of the channel. It also means that the probability that the channel can not provide the transmission rate $R$ is less than $\epsilon(R) . \epsilon$ is mainly impacted by the transmission data rate $R$ and the SNR. $R$ is determined by the modulation and coding schemes of the transmitter. SNR is determined by the transmission power and channel condition.

\section{Performance Bounds}

This section presents the results of performance bounds with traffic sources transmits data over the Rayleigh channel. We consider two cases: 1) the source periodically transmits data, which can be modeled by a deterministic arrival curve; 2) The traffic source transmits data randomly, which can be modeled by a stochastic arrival curve. Part of these derivations and proofs are based on the results in [18]. Due to space limitation, we do not present basic knowledge of network calculus. Please refer to [4] for more details.

Let $A(t)$ and $D(t)$ denote the arrival process and departure process ${ }^{1}$, respectively.

\section{A. Deterministic arrival traffic}

Theorem 1 Consider a traffic arrival process $A(t)$ bounded by a deterministic arrival curve $\alpha(t)$, and $A(t)$ receives a stochastic service curve $\langle\beta(t), \epsilon\rangle$, the performance bounds can be derived as follows.

1) Backlog bound. The stochastic backlog bound $B(t)$ is expressed as:

$$
\operatorname{Pr}\{B(t) \geq \alpha \oslash \beta(0)\} \leq \epsilon,
$$

where $\alpha \oslash \beta(0)=\sup _{t \geq 0}\{\alpha(t)-\beta(t)\}$.

\footnotetext{
${ }^{1}$ We call the input and output of the channel as arrival process and departure process, respectively.
} 
2) Delay bound. A stochastic upper bound for delay $d(t)$ is given by:

$$
\operatorname{Pr}\{d(t) \geq h(\alpha, \beta)\} \leq \epsilon,
$$

where $h(\alpha, \beta)=\sup _{t \geq 0}\{\inf [\tau \geq 0: \alpha(t) \leq \beta(t+\tau)]\}$ denotes the maximum horizontal difference between the arrival curve and service curve.

Proof: Let $t \geq s \geq 0$. From known conditions, we have $B(t)=A(t)-\bar{D}(t), \alpha \oslash \beta(0)=\sup _{t>0}\{\alpha(t)-\beta(t)\}$, $A(t)-A(s) \leq \alpha(t-s)$, and $\operatorname{Pr}\{D(t) \leq A \otimes \beta(t)\} \leq \epsilon$. Consequently,

$$
\begin{aligned}
& \operatorname{Pr}\{B(t) \geq \alpha \oslash \beta(0)\} \\
= & \operatorname{Pr}\left\{A(t)-D(t) \geq \sup _{\tau \geq 0}[\alpha(\tau)-\beta(\tau)]\right\} \\
\leq & \operatorname{Pr}\left\{A(t)-D(t) \geq \sup _{0 \leq \tau \leq t}[A(t)-A(t-\tau)-\beta(\tau)]\right\} \\
= & \operatorname{Pr}\left\{A(t)-D(t) \geq A(t)-\inf _{0 \leq \tau \leq t}[A(t-\tau)+\beta(\tau)]\right\} \\
= & \operatorname{Pr}\{D(t) \leq A \otimes \beta(t)\} \leq \epsilon .
\end{aligned}
$$

Let $\tau_{0}=h(\alpha, \beta)$. Since the delay is defined as: $d(t)=$ $\inf \{\tau \geq 0: A(t) \leq D(t+\tau)\}$, we get $\operatorname{Pr}\left\{d(t) \geq \tau_{0}\right\} \leq$ $\operatorname{Pr}\left\{A(t) \geq D\left(t+\tau_{0}\right)\right\}$. The problem becomes how to prove the bound of $\operatorname{Pr}\left\{A(t) \geq D\left(t+\tau_{0}\right)\right\}$.

$$
\begin{aligned}
& A(t)-D\left(t+\tau_{0}\right) \\
= & A(t)-A \otimes \beta\left(t+\tau_{0}\right)+A \otimes \beta\left(t+\tau_{0}\right)-D\left(t+\tau_{0}\right) \\
= & A(t)-\inf _{0 \leq s \leq t+\tau_{0}}\left[A(s)+\beta\left(t+\tau_{0}-s\right)\right] \\
& +\left[A \otimes \beta\left(t+\tau_{0}\right)-D\left(t+\tau_{0}\right)\right] .
\end{aligned}
$$

Let $V=\left[A \otimes \beta\left(t+\tau_{0}\right)-D\left(t+\tau_{0}\right)\right]$, we have

$$
\begin{aligned}
& A(t)-D\left(t+\tau_{0}\right) \\
\leq & \sup _{0 \leq s \leq t+\tau_{0}}[A(t)-A(s)-\alpha(t-s)] \\
& +\sup _{0 \leq s \leq t+\tau_{0}}\left[\alpha(t-s)-\beta\left(t+\tau_{0}-s\right)\right]+V \\
\leq & \sup _{0 \leq s \leq t+\tau_{0}}\left[\alpha(t-s)-\beta\left(t+\tau_{0}-s\right)\right]+V .
\end{aligned}
$$

Since $\tau_{0}$ is the maximum horizontal difference between $\alpha(t)$ and $\operatorname{beta}(t)$, we get $\alpha(t-s) \leq \beta\left(t-s+\tau_{0}\right)$. Therefore,

$$
\operatorname{Pr}\left\{d(t) \geq \tau_{0}\right\} \leq \operatorname{Pr}\{D(t) \leq A \otimes \beta(t)\} \leq \epsilon .
$$

\section{B. Stochastic arrival traffic}

Apart from deterministic arrival curve, an arrival process can be stochastically bounded, such as the exponentially bounded burstiness (EBB) model [19]. We consider a stochastic arrival curve model with parameters $\left(\rho, a_{1}, a_{2}\right)$ as following [13]:

$$
\operatorname{Pr}\left\{\sup _{0 \leq s \leq t}[A(t)-A(s)-\alpha(t-s)] \geq 0\right\} \leq a_{1} e^{-a_{2} \sigma}
$$

where $0 \leq s \leq t, \sigma>0$, and $\alpha(t)=\rho \cdot t+\sigma$.
Theorem 2 Consider a traffic arrival process constrained by a stochastic arrival curve, i.e., $A(t) \sim\langle\alpha(t), f(\sigma)\rangle$, where $\alpha(t)=\rho \cdot t+\sigma$ and $f(\sigma)=a_{1} e^{-a_{2} \sigma} \cdot A(t)$ receives a stochastic service curve $\langle\beta(t), \epsilon\rangle$. The performance bounds can be expressed as follows,

1) Backlog bound. A stochastic backlog bound $B(t)$ can be derived by,

$$
\operatorname{Pr}\{B(t) \geq \alpha \oslash \beta(0)\} \leq \epsilon+f(\sigma) .
$$

2) Delay bound. A stochastic upper bound for delay $d(t)$ is given by:

$$
\operatorname{Pr}\{d(t) \geq h(\alpha, \beta)\} \leq \epsilon+f(\sigma) .
$$

Since the probability can not be bigger than 1 , we define $\epsilon+f(\sigma)=\min (\epsilon+f(\sigma), 1)$.

Proof: The backlog is defined as the amount of data stored in the system. So we have,

$$
\begin{aligned}
B(t)= & A(t)-D(t) \\
= & A(t)-A \otimes \beta(t)+A \otimes \beta(t)-D(t) \\
= & A(t)-\inf _{0 \leq s \leq t}[A(s)+\beta(t-s)]+[A \otimes \beta(t)-D(t)] \\
\leq & \sup _{0 \leq s \leq t}[A(t)-A(s)-\beta(t-s)-\alpha(t-s)+\alpha(t-s)] \\
& +[A \otimes \beta(t)-D(t)] \\
\leq & \sup _{0 \leq s \leq t}[A(t)-A(s)-\alpha(t-s)]+\sup _{t \geq 0}[\alpha(t)-\beta(t)] \\
& +[A \otimes \beta(t)-D(t)] .
\end{aligned}
$$

The prove of delay bound is very similar to the case with deterministic arrival curve (Eq. (9)). The difference is in the last step of Eq. (9): for deterministic arrival curve, $\operatorname{Pr}\left\{\sup _{0<s<t}[A(t)-A(s)-\alpha(t-s)] \geq 0\right\}=0$; while for stochastic arrival curve, $\operatorname{Pr}\left\{\sup _{0 \leq s \leq t}[A(t)-A(s)-\alpha(t-\right.$ $s)] \geq 0\} \leq f(\sigma)$. Therefore, the delay bound is given by $\operatorname{Pr}\{d(t) \geq h(\alpha, \beta)\} \leq \epsilon+f(\sigma)$.

Although our work focuses on the EBB arrival model, it is straightforward to extend the method for deriving performance bounds for other types stochastic arrival curves as long as the deterministic arrival curve and violation probability are known.

\section{Performance Evaluation}

This section presents both simulation and numerical results. With simulation results, we validate the analytical approach. We study the relations between performance bounds and channel characteristics through numerical results, which also can provide hints on designing transmission strategies in wireless networks.

\section{A. Simulation results}

In order to validate the correctness and tightness of the network calculus based modeling and QoS analysis method, we conduct simulations and compare analytical results with simulation results. The parameters used in the simulation are: channel bandwidth $W=30 \mathrm{kHz}, S N R=0 \mathrm{~dB}$, packet size is $1 \mathrm{kbit}$. Each simulation is performed 50 runs 
with different seeds. In each run, the simulation period is 20000 cycles and the source generate one packet every cycle. The delay of every packet is recorded, and the backlog is recorded in every cycle. Since we can not obtain the violation probability from the simulation directly, we need to map the simulation results to delay/backlog bounds with corresponding violation probabilities. Let $m=50$ denote the total number of runs, and $n=20000$ denote the total number of packets/cycles in one simulation run. Let $d(i, j)$ $(i=1,2, \cdots, m ; j=1,2, \cdots, n)$ denote the delay of packet $j$ in simulation run $i$. We sort $d(i,:)$ in descending order, where $d(i,:)$ represents the set of values of delay in simulation run $i$. Let $\epsilon_{0}(l)=(l-1) \times \kappa(l=1,2, \cdots,[1 / \kappa\rceil)$ denote the set of violation probabilities, where $\kappa$ is a scaler (In the figures, we set $\kappa=0.05$ ). If the violation probability is $\epsilon_{0}(l)$, the corresponding delay bound is computed by $d_{0}(l)=\max _{1 \leq i \leq m}\left[d\left(i,\left\lceil n \times \epsilon_{0}(l)\right\rceil\right)\right]$. For example, the delay bound with violation probability $\epsilon_{0}(3)=0.1$ is computed as $d_{0}(3)=\max _{1 \leq i \leq m} d(i, 2000)$.

In the first simulation, the traffic source sends packet periodically with data rate $r=10 \mathrm{kbps}$ over the Rayleigh fading channel. The arrival process can be modeled by an affine arrival curve $\alpha(t)=r t+b$, with $b=1 \mathrm{kbit}$. We study and compare the analytical and simulation results of packet delays under different violation probabilities.

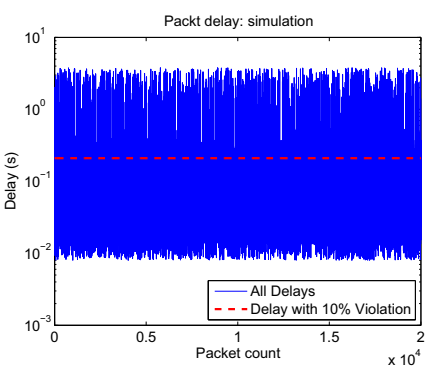

Fig. 2. Simulation results: delay.

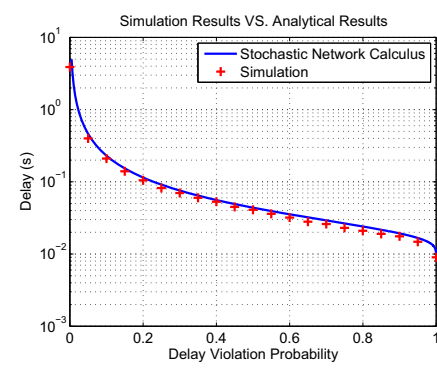

Fig. 3. Compare simulation and analytical results.
Fig. 2 illustrates the packet delays in one simulation run, where 20000 packets are sent by the source. From this figure, we can see that the packet delays are very heterogeneous, varying from $0.01 s$ to $5 s$. For the simulation data, we find that there are very few packets experience high delays. So the deterministic bound may be very loose and it is more meaningful to derive the stochastic bound. From these simulation results, we can derive the delay bounds with corresponding violation probabilities using the mapping method proposed in the previous paragraph. For example, we first sort the delay $d(i)(i=1,2, \cdots, 20000)$ in the descending order. If the violation probability is $10 \%$, the delay bound is $d(2000)$ (since $2000 / 20000=0.1$ ), which is $0.21 s$ (shown by the dashed line in Fig. 2). The delay bounds with other violation probabilties (as shown by ${ }^{\prime}+{ }^{\prime}$ marker in Fig. 3 ) can be computed similarly.

The analytical results can be computed by Eq. (6). Fig. 3 shows the comparison of analytical results and simulation results. In this figure, the maximum, average, and minimum differences between the simulation and analytical results are $5.19 \%, 9.88 \%$ and $13.5 \%$, respectively. From this figure, we can see that all the simulation results are within the bound of the analytical results. Moreover, the comparison indicates that the performance bounds derived by network calculus is reasonably tight.

Since the arrival process is constrained by a deterministic arrival curve $\alpha(t)=r t+b$, and the channel provides a stochastic rate service curve with zero processing delay, the backlog bound (Eq. (5)) is only determined by the burstiness of the input. Hence, the analytical backlog bound is constant and independent of the violation probability. In this case, we need use empirical method to derive an empirical backlog bound by combining the analytical and simulation results.

Simulation with Stochastic Arrival Traffic: In the second simulation, the traffic arrival process is a stationary two-state Markov process $A(t), t=(i-1) t_{0}, i=1,2,3, \cdots\left(t_{0}\right.$ is the inter-arrival time) with transition probabilities $p_{12}=10^{-6}$ and $p_{21}=10^{-2}$. When in state 1 , the source is generating $n_{0}$ packet with probability $1 / 8$ and no packet with probability $7 / 8$. When in state 2 , the source is generating $n_{0}$ packet with probability $9 / 20$ and no packet with probability $11 / 20$. The packet size is $1 \mathrm{kbit}$. This process can be characterized by the EBB arrival model as following, $\operatorname{Pr}\{\sup [A(t)-A(s)] \geq \rho(t-s)+\sigma\} \leq \exp \left(-a_{2} \sigma\right)$, where $a_{2}=2.73 e-3$ and $0 \leq s \leq t[20]$.

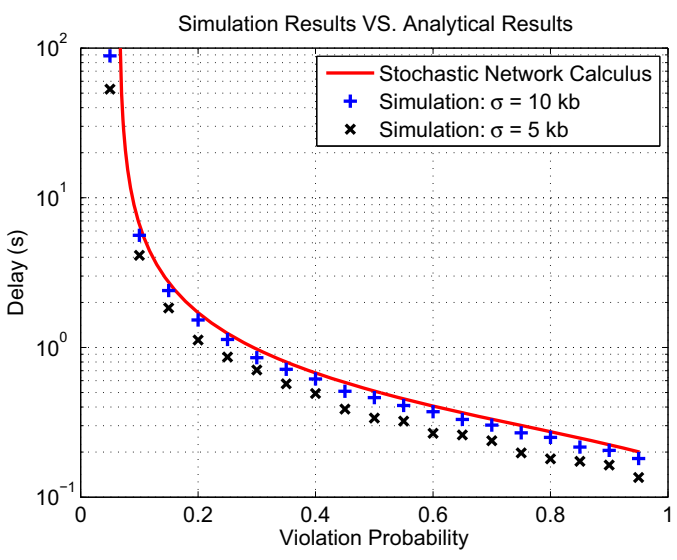

Fig. 4. Compare simulation results with analytical results: Markov arrivals.

Fig. 4 shows the comparison of analytical results (by network calculus) and simulation results. For the analytical results, we set input burstiness $10 \mathrm{kbit}$. We implement two simulations with input data rate $\rho=10 \mathrm{kbps}$, and burstiness $\sigma$ equals to $5 \mathrm{kbit}$ and $10 \mathrm{kbit}$, respectively. The input burstiness denotes the number of packets that the source generate at a time. For example, $\sigma=5$ corresponds to $n_{0}=5$ and $t_{0}=0.5 \mathrm{~s}$, and $\sigma=10$ corresponds to $n_{0}=10$ and $t_{0}=1 \mathrm{~s}$. From the comparison, we can see that both simulation results are within the bounds of analytical results, which validate the correctness of the analytical method. Furthermore, with the same setting of input burstiness, i.e., $\sigma=10 \mathrm{kbit}$, the simulation and analysis results are very close to each other. The 
maximum, average, and minimum differences between them are $8.25 \%, 10.5 \%$ and $15.8 \%$, respectively. The comparison indicates that the tightness of delay bound derived by network calculus is good.

\section{B. Numerical results}

In the first experiment, we study the performance bounds under the case of a periodic traffic source sending packets over the Rayleigh fading channel. The traffic input process is modeled an affine arrival curve [4], which is defined as $\alpha(t)=r t+b$, where $b$ and $r$ represent the burst tolerance (in bits) and the average data rate (in bit/s), respectively. In this experiment, we set $b=1 \mathrm{kbit}$ and $r=10 \mathrm{kbps}$.

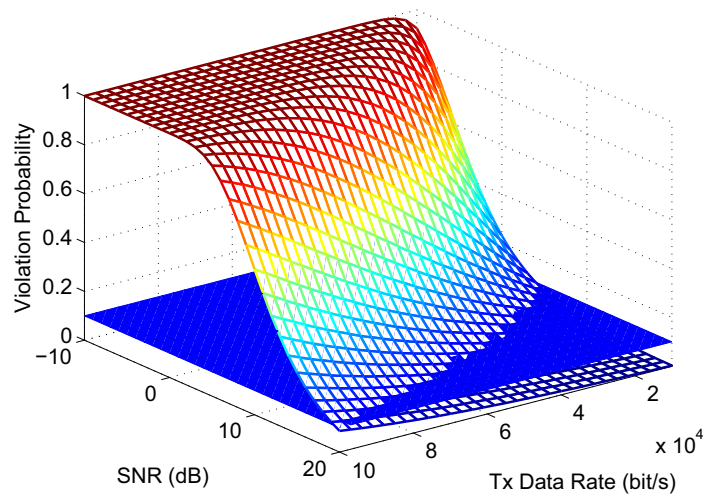

Fig. 5. Delay bound under different SNR and transmission data rates.

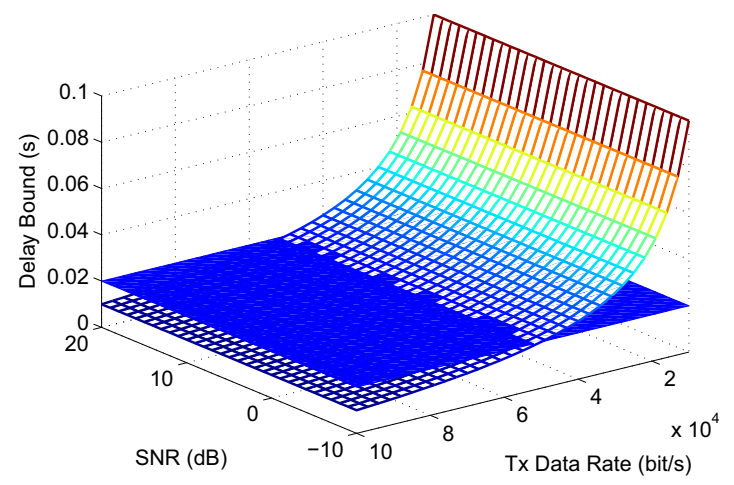

Fig. 6. Violation probability of the delay and backlog bounds.

If $R>r^{2}$, the delay bound can be calculated by $h(\alpha, \beta)=$ $b / R$, and the backlog bound is $\alpha \oslash \beta(0)=b$. Fig. 5 shows the values of delay bound varying with $R \in[10 \mathrm{kbps}, 100 \mathrm{kbps}]$ and $S N R \in[-10 d B, 20 d B]$. The delay bound $d$ is determined by the input burstiness $b$ and the transmission data rate $R$. From Fig. 5, we can see that with a fixed $b$, it increases as transmission rate increases, and it is independent

\footnotetext{
${ }^{2}$ If $R<r$, both the delay and backlog would increase with time. It is impossible and meaningless to find the finite delay and backlog bounds. Therefore, we consider the case that $R \geq r$.
}

of the SNR. The backlog bound is only determined by the burstiness of the input traffic. However, the corresponding violation probabilities of these two bounds are impacted both by the transmission data rate and SNR (as shown in Fig. 6). The violation probability increases as transmission data rate increases and/or SNR decreases. There is a trade-off between the delay bound and its violation probability. If we want to reduce the transmission delay, a high transmission rate should be chosen. But high transmission rate brings high violation probability.

The analysis method not only can be used to derive performance bounds and their corresponding violation probabilities, but also can provide guidelines for designing modulation and coding schemes, i.e., the designer can choose appropriate transmission strategies according to performance requirements. For example, if the performance requirement is given as: $d<0.02$ ( as shown by the plane in Fig. 5) with violation probability less than $10 \%$ (as shown by the plane in Fig. 6), we can get the following system configurations: $R>50 \mathrm{kbps}$ and $S N R>14 d B$, which are the values constraining delay and violation probability within the threshold. Hence, the designer can choose corresponding transmission strategy (transmission power, modulation and coding schemes) according to $R$ and $S N R$.

Numerical Results with Stochastic Arrival Traffic: In the following experiments, we study the performance bounds under the case of a random traffic source sending packets over the Rayleigh fading channel. The traffic input process is modeled by the EBB arrival curve (Eq. (11)). The values of parameters are: $a_{1}=1, a_{2}=1 e-3$, and $\rho=10 \mathrm{kbps}$. Similar to the previous experiment, we assume $\rho<R$.

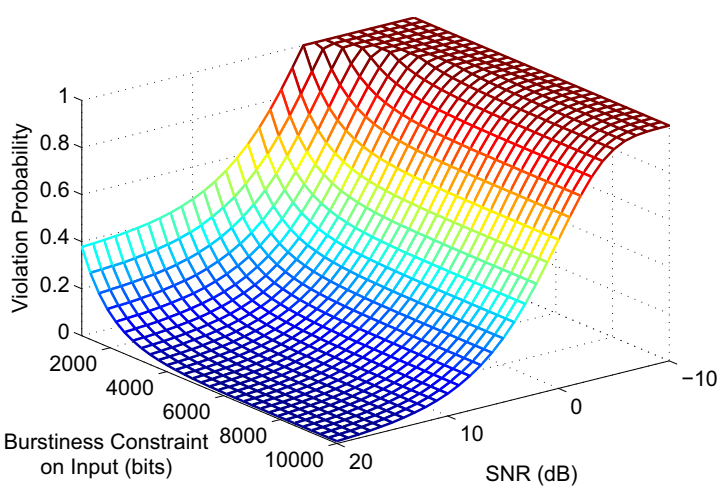

Fig. 7. Violation probability of the delay and backlog bounds.

In this experiment, we fix the transmission data rate $R=$ $30 \mathrm{kbps}$ and change the values of input burstiness $(\sigma)$ and SNR. Both the delay $(d=\sigma / R)$ and backlog $(B=\sigma)$ bounds are linearly increasing with input burstiness. Their corresponding violation probabilities are illustrated in Fig. 7, where we can see that the violation probabilities decrease as $S N R$ and/or $\sigma$ increase. Hence, there are trade-offs between performance bounds and their violation probabilities. High $\sigma$ would cause low violation probability, but bring high delay 
and backlog. Therefore, appropriate values of $\sigma$ and $S N R$ should be chosen according to the performance requirements.

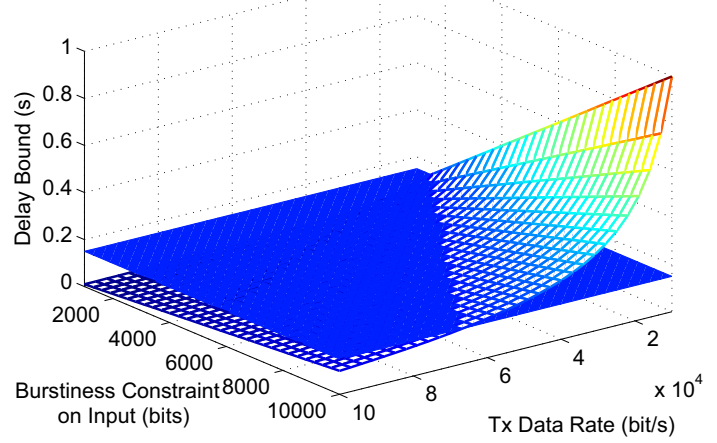

Fig. 8. The delay bound.

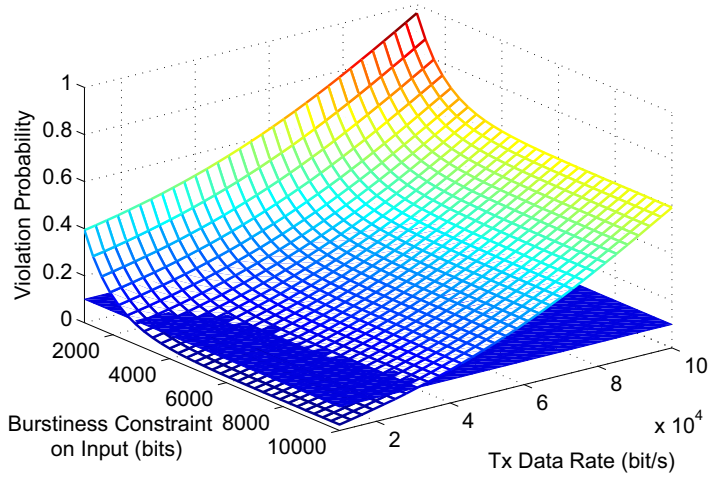

Fig. 9. Violation probability of the delay and backlog bounds.

In this experiment, we fix the $S N R=10 d B$ and change the values of input burstiness $(\sigma)$ and transmission data rate $R$. Similarly, the backlog bound is only determined by $\sigma$. The delay bound is shown in Fig. 8. The violation probabilities of these two bounds are illustrated in Fig. 9.

From Fig. 8, we can see that the delay bound increases as input burstiness increases, and it decreases as transmission data rate increases. While the violation probability of the bounds has an opposite behavior, i.e., it decreases as the input burstiness increases and it increases as the transmission data rate increases. Moreover, from these performance results we can derive the system configurations which can meet the requirements. For example, if the performance requirements are defined as: $d<0.15 \mathrm{~s}, B<5 \mathrm{kbit}$ with violation probability less than $10 \%$, then we should select the values of $S N R$ and $\sigma$ below the planes in Fig. 8 and Fig. 9.

\section{CONCLUSION}

In this paper, we propose a method for modeling and QoS analysis of wireless channels subject to Rayleigh fading. The key challenge in analyzing wireless systems is the temporal uncertainties inherent in fading channels. To this end this work applies stochastic network calculus to model Rayleigh fading channels and derive stochastic delay and backlog bounds. The analysis method is validated through simulations. Moreover, numerical experiments show that the performance results can be used to provide guidelines for design transmission strategies. Our future work would focus on modeling wireless channels with other types of fading.

\section{REFERENCES}

[1] R. Cruz. A calculus for network delay, part I: Network elements in isolation. IEEE Transaction on Information Theory, 37(1):114-121, 1991.

[2] R. Cruz. A calculus for network delay, part II: Network analysis. IEEE Transaction on Information Theory, 37(1):132-141, 1991.

[3] C. S. Chang. Performance Guarantees in Communication Networks. Springer-Verlag, 2000.

[4] J. Boudec and P. Thiran. Network calculus: A theory of deterministic queuing systems for the internet. Springer, LNCS 2050, 2004.

[5] Yuming Jiang. A basic stochastic network calculus. in Proceeding of ACM SIGCOMM, 2006.

[6] Jens B. Schmitt and U. Roedig. Sensor network calculus - a framework for worst case analysis. IEEE/ACM Int. conference on Distributed Computing in Sensor Systems (DCOSS'05), 2005.

[7] Anis Koubaa, M. Alves, and E. Tovar. Modeling and worst-case dimensioning of cluster-tree wireless sensor networks. The 27th IEEE International Real-time Systems Symposium (RTSS'06), 2006.

[8] Jens B. Schmitt, F. A. Zdarsky, and L. Thiele. A comprehensive worstcase calculus for wireless sensor networks with in-network processing. In Proceedings of the 28th IEEE Int. Real-time Systems Symposium (RTSS'07), 2007.

[9] Y. Cao, Y. Xue, and Y. Cui. Network-calculus-based analysis of power management in video sensor networks. the IEEE Global Communications Conference, Exhibition and Industry Forum (GLOBECOM'07), 2007.

[10] Petr Jurcik, Ricardo Severino, Anis Koubaa, Mario Alves, and Eduardo Tovar. Real-time communications over cluster-tree sensor networks with mobile sink behaviour. In 14th IEEE International Conference on Embedded and Real-Time Computing Systems and Applications (RTCSA'08), August 2008.

[11] Huimin She, Zhonghai Lu, Axel Jantsch, Dian Zhou, and Li-Rong Zheng. Analytical evaluation of retransmission schemes in wireless sensor networks. In Proc. of IEEE 69th Vehicular Technology Conference (VTC2009-Spring), 2009.

[12] Florin Ciucu, Almut Burchard, and Jörg Liebeherr. A network service curve approach for the stochastic analysis of networks. In Proceedings of the 2005 ACM SIGMETRICS international conference on Measurement and modeling of computer systems (SIGMETRICS'05), pages 279-290, 2005.

[13] A. Burchard, J. Liebeherr, and S. D. Patek. A min-plus calculus for endto-end statistical service guarantees. IEEE Transactions on Information Theory, 52(9):4105-4114, Sept. 2006.

[14] Yuming Jiang, Qinghe Yin, Yong Liu, and Shengming Jiang. Fundamental calculus on generalized stochastically bounded bursty traffic for communication networks. Computer Networks, 53(12):2011-2021, 2009.

[15] G. Verticale and P. Giacomazzi. An analytical expression for service curves of fading channels. In IEEE Global Telecommunications Conference (GLOBECOM'09), pages $1-6$, Nov. 2009.

[16] Yuming Jiang and Peder J. Emstad. Analysis of stochastic service guarantees in communication networks: A server model. In In Proceeding of the International Workshop on Quality of Service (IWQoS'05), pages 233-245, 2005.

[17] Theodore S. Rappaport. Wireless Communications: Principles and Practice. Prentice Hall, 2002.

[18] Yuming Jiang and Yong Liu. Stochastic Network Calculus. Springer Publishing Company, 2008.

[19] Opher Yaron and Moshe Sidi. Performance and stability of communication networks via robust exponential bounds. IEEE/ACM Transaction on Networking, 1(3):372-385, 1993.

[20] David Starobinski and Moshe Sidi. Stochastically bounded burstiness for communication networks. IEEE Transaction on Information Theory, 46(1):206-212, 2000. 